Check for updates

Cite this: RSC Adv., 2018, 8, 26461

\title{
Self-assembly of peptide amphiphiles by vapor pressure osmometry and dissipative particle dynamics
}

\author{
Taiga Seki, ${ }^{a}$ Noriyoshi Arai, (D) *bc Donguk Suh, ${ }^{d}$ Taku Ozawa, ${ }^{e}$ Tomoko Shimada, ${ }^{f}$ \\ Kenji Yasuoka ${ }^{a}$ and Atsushi Hotta $(\mathbb{D}$ *a
}

Peptide amphiphiles are one of the most promising materials in the biomedical field, so much effort has been devoted to characterizing the mechanism of their self-assembly and thermosensitive gelation. In this work, vapor pressure osmometry measurements were carried out to parameterize the thermosensitivity of interactions between peptide amphiphiles in an aqueous solution. The osmometry measurement verified that the peptides became more hydrophobic as temperature increased, which was quantitatively described with the Flory-Huggins $\chi$ parameter. Thereafter, a coarse-grained molecular model was used to simulate peptide amphiphiles dissolved in an aqueous solution. The temperature sensitive coarse-grained parameter $a_{H W}$, which is the repulsive force between the hydrophilic head of the peptide amphiphile and water was estimated from the aforementioned experimentally obtained $\chi$. Furthermore, the effects of concentration and temperature on the self-assembly behavior of peptide amphiphiles were quantitatively studied by dissipative particle dynamics. The simulation results revealed that $a_{\mathrm{HW}}$ plays an important role in self-assembly characteristics and in the resulting microstructure of the peptide amphiphiles, which coincides with previous experimental and computational findings. The methodology in quantitatively linking the coarse-grained parameter from experiment and theory provides a sensible foundation for bridging future simulation studies with experimental work on macromolecules.

Received 1st June 2018

Accepted 16th July 2018

DOI: $10.1039 / c 8 r a 04692 a$

rsc.li/rsc-advances

\section{Introduction}

Stimuli-responsive polymers are known to change their macroscopic properties based on variations in external conditions such as temperature, $\mathrm{pH}$, light, and magnetic fields. Because of their unique nature, polymers have gathered great interest in academia and industry. ${ }^{1}$ Polymers exhibiting temperatureinduced gelation characteristics have specifically attracted significant interest in the biological and biomedical fields. ${ }^{2}$ There are numerous reports on thermosensitive polymers having been created from poly(ethylene glycol), ${ }^{3}$ poly(ethylene oxide), ${ }^{4}$ and poly( $N$-isopropylacrylamide). ${ }^{\mathbf{1 , 5 , 6}}$

\footnotetext{
${ }^{a}$ Department of Mechanical Engineering, Keio University, 3-14-1 Hiyoshi, Kohoku-ku, Yokohama 223-8522, Japan. E-mail: hotta@mech.keio.ac.jp

${ }^{b}$ Department of Mechanical Engineering, Kindai University, 3-4-1 Kowakae, Higashiosaka, Osaka 577-8522, Japan

${ }^{c}$ Research Institute for Science and Technology, Kindai University, 3-4-1 Kowakae, Higashiosaka, Osaka 577-8522, Japan.E-mail: arai@mech.kindai.ac.jp

${ }^{d}$ Department of Mechanical Engineering, University of Tokyo, 7-3-1 Hongo, Bunkyo-ku, Tokyo 113-8656, Japan

${ }^{e}$ Materials Science Section, Engineering Technology Division, JSOL Corporation, Harumi Center Bldg., 2-5-24, Harumi, Chuo-ku, Tokyo 104-0053, Japan

${ }^{f}$ Asahi-Kasei Corporation, 1-105 Jimbocho, Kanda, Chiyoda-ku, Tokyo 100-8101, Japan
}

Peptide amphiphiles (PA) have also attracted attention as
nother thermosensitive material. ${ }^{7,8}$ Amphiphilic molecules self-assemble to form a highly ordered nanoscale configuration in the presence of a solvent. Though general amphiphilic molecules lose their self-assembling ability and disperse into the solvent as temperature rises, some PAs have been found to exhibit temperature-induced gelation characteristics. ${ }^{\mathbf{9} 10}$ The amphiphiles also show excellent biocompatibility and biodegradability, making them one of the most prospective materials for drug delivery systems, ${ }^{11}$ scaffolds for tissue engineering, ${ }^{12}$ regenerative medicine, ${ }^{\mathbf{1 3}}$ and other "tailor-made" materials for biomedical use. ${ }^{14}$

Besides exploring new sequences to inject functionality into PA for particular applications, considerable effort has been put into understanding the self-assembly mechanism. Several studies have revealed that the self-assembly mechanism of PA is more complicated compared to conventional amphiphilic molecules because their assembly behavior is extensively affected by the secondary structure of $\alpha$-helix and $\beta$-sheet transitions. ${ }^{12,15-18}$ Nowak et al. ${ }^{12}$ employed rheological and morphological analyses on PA with several peptide sequences and found that gelation was tied to the conformation of the hydrophobic peptide domains. They reported that the $\alpha$-helical segments worked as good gelators, followed by the $\beta$-sheet and 
then the random coils. Such simultaneous phase transitions were also reported by Ding et al. ${ }^{15}$ which had conducted both experimental observations and a coarse-grained molecular dynamics simulation using a simulator called COGNAC, ${ }^{19}$ to confirm that the self-assembly transition from an $\alpha$-helix to a random coil would cause a change in the micelle structure from a worm to a sphere. Furthermore, Lee et al. ${ }^{\mathbf{1 6}}$ conducted an atomistic molecular dynamics simulation on PA to construct nanofibers and found that water and ions could still penetrate the outer core region even after the nanostructure became matured. Lee et $a l .{ }^{17}$ also used a coarse-grained force field called MARTINI $^{20}$ to perform simulations on PAs as long as a few microseconds and visualized the three-dimensional network transforming into a quasi-one-dimensional nanofiber. Fu et $a l .{ }^{18}$ used ePRIME, ${ }^{21}$ which is an extended PRIME model, ${ }^{22}$ as their coarse-grained model to simulate and adjusted the hydrophobicity to observe the various configurational transitions from open structures to a $\beta$-sheet and/or random coils.

We have worked with a particular PA named $\mathrm{C}_{16}-\mathrm{WA}_{4} \mathrm{KA}_{4}$ $\mathrm{KA}_{4} \mathrm{KA}$ (hereafter $\mathrm{C} 16-\mathrm{W} 3 \mathrm{~K}$ ), which consists of a hydrophobic alkyl chain and hydrophilic peptides containing tryptophan $(\mathrm{W})$, lysine (K), and alanine (A). ${ }^{23-25}$ Their aqueous solution revealed that the self-assembly structure transition from a spherical to a worm-like micelle and peptide conformation transitions from $\alpha$-helix to $\beta$-sheet simultaneously took place with macroscopic sol-gel transitions, which was found to be temperature-sensitive. The reproduction of the experiments took nearly a month at room temperature but was significantly accelerated at $50{ }^{\circ} \mathrm{C}$, where the product could be made in 90 minutes. It was also noteworthy that the worm-like micelle structure could be maintained even in dry conditions, whereas self-assembled structures of other general amphiphilic molecules were produced only in the presence of a solvent. Such high stability of the worm-like micelle should be largely due to the contribution of the intermolecular hydrogen bonds of the $\beta$ sheets. The precise mechanism of the phase transitions of C16W3K has yet to be elucidated, but Zhou et al. ${ }^{4}$ explained that the source of thermosensitivity of hyperbranched poly(3-ethyl-3oxetanemethanol)-poly(ethylene oxide) (HBPO-star-PEO) was from the hydrogen bonding ability of a PEO segment weakening as temperature rose, leading to a partial collapse of the hydration shell around the aggregates. The disruption of the hydration shell induced the collision and fusion of the aggregates resulting in a morphological transformation. Besides the previous experimental studies, Duce et $a .^{26}$ found that interpeptide hydrogen bonds limited the diffusivity and that the conformation eventually determined the morphology of the PA aggregates analyzed by molecular simulations. The concern with the molecular simulations, especially with the coarsegrained simulations, should be in establishing a scientific basis for the interaction parameters to support the findings.

In this study, we used experimental measurements, where we applied the Flory-Huggins theory to extract the interaction parameter for our coarse-grained model and applied it to dissipative particle dynamics (DPD) simulations. ${ }^{27,28}$ DPD is known to directly allocate molecular information to physical parameters such as the Flory-Huggins $\chi$ parameter, ${ }^{29}$ and numerous examples on the self-assembly of amphiphiles were successfully reproduced by this simulation method. ${ }^{30-35}$ Jury et al. ${ }^{32}$ constructed a coarse-grained model for C12E6, which is a non-ionic amphiphile, and produced a temperatureconcentration phase diagram that was consistent with experiments. Nakamura et al. ${ }^{35}$ used the aforementioned C12E6 model and studied how the change in the interaction between the hydrophilic part and solution would affect the temperatureconcentration phase diagram, whereas Arai et al. ${ }^{33}$ investigated the self-assembly dynamic mechanism of the configurational transformation from spherical to worm-like micelles.

There is still, however, limited work regarding the temperature effects on the self-assembly of PA using DPD, so both experimental and computational methods were used to further investigate our previous report on temperature-sensitive selfassembly of PA. ${ }^{23}$ The ensuing section will introduce experimental and simulation theories applied to the analysis of this study. The conditions for both methods will be explained, which will be followed by the results and discussion of this work.

\section{Theory}

\subsection{Vapor pressure osmometry}

Vapor pressure osmometry (VPO) measurements were used to calculate $\chi$ between the peptide segments and water. VPO is a quick and convenient method to measure thermodynamic properties for dilute and semi-dilute solutions of polymer with low molecular weight. ${ }^{36,37}$ The apparatus is composed of two thermistors that form two arms of a Wheatstone bridge in an enclosed measuring chamber. The temperature is carefully controlled so that the chamber is saturated with solvent vapor. Under these conditions, the pressure difference between a pure solvent and solution is obtained from the voltage (temperature) differences between the two thermistors when a drop of pure solvent and solution comes into contact with each thermistor. The measured voltage difference has the following relationship with the osmotic pressure $\Pi$ and temperature $T$ :

$$
\frac{\Pi}{R T}=\frac{\Delta \Phi_{\mathrm{el}} \rho_{1}}{1000 K},
$$

where $\Delta \Phi_{\mathrm{el}}$ is the voltage difference, $\rho_{1}$ is the density of solvent, $R$ is the gas constant, and $K$ is a calibration constant. The chemical potential related to the solvent activity in the solution and the osmotic pressure is:

$$
\Delta \mu_{1}=R T \ln a_{1}=-\nu_{1} \Pi .
$$

According to the Flory-Huggins theory, ${ }^{38}$ the interaction between a solvent and solute can be calculated from

$$
\frac{\Delta \mu_{1}}{R T}=\ln \phi_{1}+\left(1-\nu_{1} / \nu_{2}\right) \phi_{1}+\chi_{12} \phi_{2}^{2}
$$

where $\nu_{1} / \nu_{2} \approx 0$ is the ratio of the molar volumes of the solvent and polymer. Moreover, the volume fractions of the solvent and polymer are denoted by $\phi_{1}$ and $\phi_{2}$, respectively. This equation could be rewritten as: 


$$
\frac{\ln \left(a_{1} / \phi_{1}\right)-\phi_{2}}{\phi_{2}}=\chi_{12} \phi_{2}
$$

If the left-hand side of eqn (4) is plotted against $\phi_{2}$, the solvent/solute interaction parameter $\chi_{12}$ can be obtained from the slope of the fitted line.

\subsection{Dissipative particle dynamics}

The DPD method ${ }^{27,29}$ is based on Newton's equation of motion for a particle $i$,

$$
m_{i} \frac{\mathrm{d} \mathbf{v}_{i}}{\mathrm{~d} t}=\mathbf{f}_{i}=\sum_{j \neq i} \mathbf{F}_{i j}^{\mathrm{C}}+\sum_{j \neq i} \mathbf{F}_{i j}^{\mathrm{D}}+\sum_{j \neq i} \mathbf{F}_{i j}^{\mathrm{R}}
$$

where $m$ is the mass, $\mathbf{v}$ is the velocity, $\mathbf{f}$ is the total force and $\mathbf{F}^{\mathbf{C}}$, $\mathbf{F}^{\mathrm{D}}, \mathbf{F}^{\mathrm{R}}$ are the conservative, dissipative, and random forces, respectively, each of which is given by:

$$
\begin{gathered}
\mathbf{F}_{i j}^{\mathrm{C}}=\left\{\begin{array}{cc}
a_{i j}\left(1-\frac{\left|\mathbf{r}_{i j}\right|}{r_{\mathrm{C}}}\right) \mathbf{n}_{i j}, & \left|\mathbf{r}_{i j}\right| \leq r_{\mathrm{C}} \\
0, & \left|\mathbf{r}_{i j}\right|>r_{\mathrm{C}}
\end{array}\right. \\
\mathbf{F}_{i j}^{\mathrm{D}}=\left\{\begin{array}{cc}
-\gamma \omega^{\mathrm{D}}\left(\left|\mathbf{r}_{i j}\right|\right)\left(\mathbf{n}_{i j} \cdot v_{i j}\right) \mathbf{n}_{i j}, & \left|\mathbf{r}_{i j}\right| \leq r_{\mathrm{C}} \\
0, & \left|\mathbf{r}_{i j}\right|>r_{\mathrm{C}}
\end{array}\right. \\
\mathbf{F}_{i j}^{\mathrm{R}}=\left\{\begin{array}{cc}
\sigma \omega^{\mathrm{R}}\left(\left|\mathbf{r}_{i j}\right|\right) \zeta_{i j} \Delta t^{-1 / 2} \mathbf{n}_{i j}, & \left|\mathbf{r}_{i j}\right| \leq r_{\mathrm{C}} \\
0, & \left|\mathbf{r}_{i j}\right|>r_{\mathrm{C}}
\end{array}\right.
\end{gathered}
$$

where $a_{i j}$ is the repulsion parameter between particles $i$ and $j$. Additionally, $r_{\mathrm{C}}$ is the cutoff distance, $\sigma$ and $\gamma$ are the noise and friction parameters, respectively, $\xi$ is a random fluctuating variable, and finally, $\omega^{\mathrm{R}}$ and $\omega^{\mathrm{D}}$ are weight functions that depend on $r=\left|\mathbf{r}_{i j}\right|$. Moreover, $\mathbf{v}_{i j}=\mathbf{v}_{i}-\mathbf{v}_{j}, \mathbf{n}_{i j}=\mathbf{r}_{i j} /\left|\mathbf{r}_{i j}\right|$ and $\omega^{\mathrm{R}}$, $\omega^{\mathrm{D}}$, as well as $\sigma$ and $\gamma$ have the following relations ${ }^{39}$

$$
\begin{gathered}
\omega^{\mathrm{D}}(r)=\left[\omega^{\mathrm{R}}(r)\right]^{2}=\left\{\begin{array}{cc}
1-\frac{\left|\mathbf{r}_{i j}\right|}{r_{\mathrm{C}}}, & \left|\mathbf{r}_{i j}\right| \leq r_{\mathrm{C}} \\
0, & \left|\mathbf{r}_{i j}\right|>r_{\mathrm{C}}
\end{array}\right. \\
\sigma^{2}=2 \gamma k_{\mathrm{B}} T
\end{gathered}
$$

In addition, a spring force $\mathbf{F}^{\mathrm{S}}$ shown below for the bond between DPD particles in PA is applied:

$$
\mathbf{F}_{i j}^{\mathrm{S}}=-C\left(1-\frac{\left|\mathbf{r}_{i j}\right|}{r_{\mathrm{S}}}\right) \mathbf{n}_{i j},
$$

where $r_{\mathrm{S}}$ is the equilibrium bond distance and $C$ is the spring constant.

\section{Setup conditions}

\subsection{Experiment}

3.1.1. Preparation of the PA solution. Peptide W3K was synthesized by Scrum Inc. and treated with trifluoroacetic acid and piperidine to remove BOC- and FMOC-groups. ${ }^{\mathbf{4 0 , 4 1}}$ The deprotected peptides were purified and freeze-dried before solubilizing in a distilled water at a concentration of $\sim 1-2 \mathrm{wt} \%$.
The characteristics of the deprotected peptides were verified by MALDI-TOF mass spectrometry.

3.1.2. VPO measurement method. A vapor pressure osmometer manufactured by Knauer was used to measure the activity of water in the aqueous peptide solutions. The measuring chamber was filled with approximately $20 \mathrm{ml}$ of pure solvent and then equilibrated at specific temperatures for a minimum of 8 hours for each experiment. Before the measurements, $\mathrm{NaCl}$ (Sigma-Aldrich Japan) was applied to calibrate the instrument. The $\Delta \Phi_{\mathrm{el}}$ values obtained for four different $\mathrm{NaCl}$ aqueous solutions (0.2-1 wt\%) were separated by concentration before being plotted. The data points were linearly fit to acquire the calibration constant $K$ in eqn (1), which was an extrapolated value of $\Delta \Phi_{\mathrm{el}} / c$, where $c$ is the concentration. Finally, the estimated $K$ values were 161 at $40{ }^{\circ} \mathrm{C}, 173$ at $50{ }^{\circ} \mathrm{C}$, and 194 at $60{ }^{\circ} \mathrm{C}$.

The measurements for the aqueous peptide systems were performed for three different concentrated solutions ranging from 1 to $2 \mathrm{wt} \%$. The temperature was varied from $40{ }^{\circ} \mathrm{C}$ to $60{ }^{\circ} \mathrm{C}$ because the manufacturer suggested the experiments to be carried out at a temperature that is at least $15{ }^{\circ} \mathrm{C}$ higher than room temperature for better stability and accuracy of the experiments.

\subsection{Simulation method}

The velocity Verlet algorithm was used to integrate the stochastic equations of motion into DPD $^{29}$ through the OCTA platform. ${ }^{19,42}$ The PA model consists of nine bead-chain particles, where the first six in sequence had hydrophilic parameters representing the peptide head and the latter three represented hydrophobic tails that mimicked the alkyl chains as in Fig. 1. Groot and Warren ${ }^{29}$ connected the Flory-Huggins theory with DPD for a system with particle number $2<N<10$ and $N_{\mathrm{A}}=N_{\mathrm{B}}$, but there are numerous reports on variants that were also confirmed experimentally. ${ }^{43-45}$ In this system, the $N_{\mathrm{A}}: N_{\mathrm{B}}$ ratio was determined from examining the actual length of peptide amphiphiles, where the head and tail had an actual length ratio of around $2: 1$; therefore, $6: 3$ was chosen because it falls within $2<N<10$ and can reproduce the flexibility of the PA. The $a_{i j}$ parameters in eqn (6) were decided from Yamamoto and Hyodo. ${ }^{46}$ The peptide-water interaction parameter $a_{\mathrm{Hw}}$ was varied from 10 to 30 to describe the change in the hydration force, where the repulsive hydration force between hydrophilic peptides decreased as temperature increased. ${ }^{4}$ Other interaction parameters were predetermined to be $a_{\mathrm{HH}}=a_{\mathrm{TT}}=a_{\mathrm{WW}}=$ $25, a_{\mathrm{HT}}=40$, and $a_{\mathrm{TW}}=80$, where $\mathrm{W}, \mathrm{H}$, and $\mathrm{T}$ denoted water, hydrophilic head, and hydrophobic tail, respectively. The total number of particles was constant at 18000 and the volume fraction (i.e. the ratio of the number of particles) of the PA molecules was changed from $10 \%$ to $50 \%$. For lower volume fractions of PA at $2 \%$ or $5 \%$, larger systems of 135000 and 72000 particles were simulated, respectively, so there was always a sufficient amount of PA molecules within the system (Table 1). The basis of the number of particles was from test cases that found the worm-like micelle to be stable when it consists of around 200 chains (PA molecules). This is consistent 

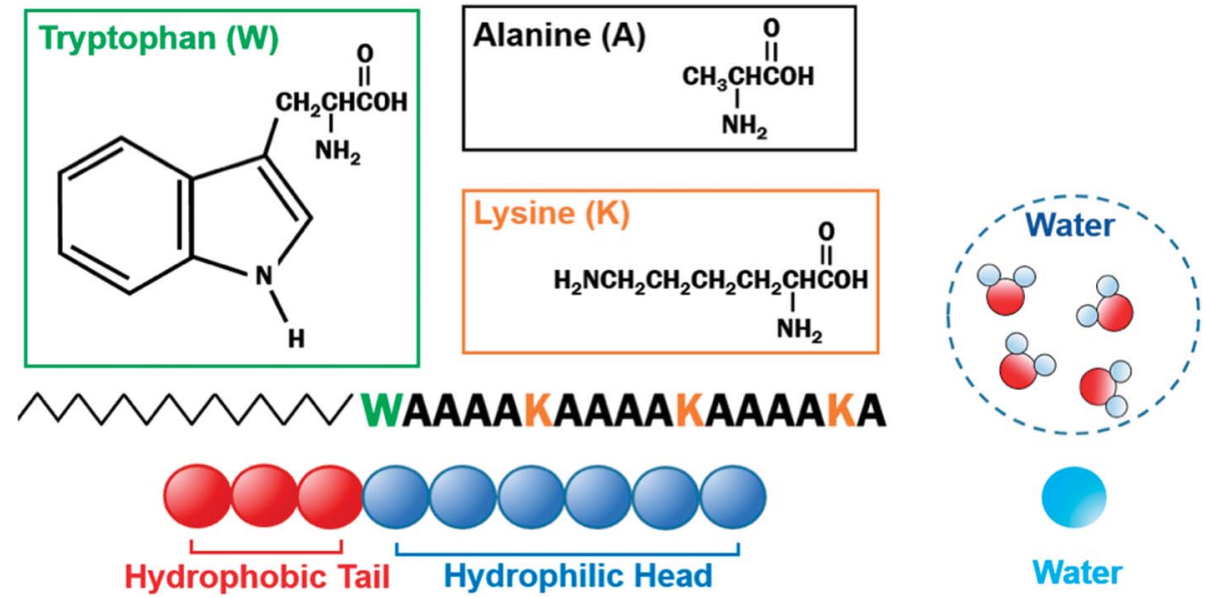

Water

Fig. 1 Schematic of coarse-grained C16-W3K and water. C16-W3K consists of an alkyl chain, which has a hydrophobic and a hydrophilic part. Three beads were used to represent the former, whereas six beads were incorporated for the latter. Four water molecules are described by a single bead.

with Arai et al., ${ }^{33}$ where the number of molecules that form worm-like micelles were found to be around 160. Calculations were carried out for 500000 steps at $\Delta t=0.05 \tau$, where $\tau$ is unit dimensionless time. During all simulations, the particle density $\rho$ was 3.0 and the volume of the simulation box in dimensionless units was $18.1712^{3}$ for $10-50 \%, 28.8450^{3}$ for $5 \%$, and $35.5689^{3}$ for $2 \%$. The equilibrium bond distance $r_{\mathrm{S}}$ between particles in PA was set to 0.86 and the spring constant $C$ was 4 based on earlier studies. ${ }^{47-51}$ Here, 0.86 corresponds to the average distance of the nearest neighbor particles. The effect of periodic boundary conditions has been examined in a separate study by Arai et al..$^{48}$ Under the current system sizes that were examined, the percolation characteristics showed no change in the self-assembly configuration and minimal variation in the $a_{\mathrm{HW}}$ value. Equilibration was determined by comparing the results from 500000 steps of four independent simulations from different initial coordinates. The results presented are only those that have produced the same terminal states.

\section{Results and discussion}

\subsection{VPO measurements}

The solvent activities were measured by VPO for the aqueous solutions of the peptides at concentrations of 1-2 wt $\%$. The experimental results were plotted in Fig. 2 using eqn (4) from the Flory-Huggins theory. The volume fraction of peptides $\phi$ was $2900 \mathrm{ml} \mathrm{mol}^{-1}$ from density measurements. A linear fit was used in the VPO measurements, which was inserted into eqn (4). The corresponding $\chi$ values were 1.10 at $40{ }^{\circ} \mathrm{C}, 1.35$ at $50{ }^{\circ} \mathrm{C}$, and 1.52 at $60{ }^{\circ} \mathrm{C}$, indicating that the interaction between the peptides and water became more repulsive as the temperature rose. Based on these results, the temperature dependence of $\chi$ for the peptides was found. Furthermore, the Flory-Huggins $\chi$ parameter is also known to have a relation with the interaction parameter $a_{i j}$ as in eqn (26) and (27) of ref. 29.

$$
\begin{aligned}
& a_{i j}(T)=a_{i i}+\Delta a(T) \\
& \Delta a(T) \approx 3.268 \chi(T)
\end{aligned}
$$

The $a_{\mathrm{HW}}$ value is calculated from eqn (12) by inserting 25 for $a_{i i}$ and the aforementioned $\chi$ values providing a maximum of $a_{\mathrm{HW}}=30$, which is used in the simulations. A $10{ }^{\circ} \mathrm{C}$ difference produces an approximately 0.65 variation in $a_{\mathrm{HW}}$.

\subsection{DPD simulations}

The phase diagram with concentration and the $a_{\mathrm{HW}}$ parameter is plotted in Fig. 3. As the concentration of PA and $a_{\mathrm{HW}}$ increased, a transition in the self-assembly structures changed

Table 1 Concentration, number of particles, and box size in DPD simulation

\begin{tabular}{llll}
\hline Concentration [\%] & $\begin{array}{l}\text { Total number } \\
\text { of particles }\end{array}$ & $\begin{array}{l}\text { Number of PA } \\
\text { molecules }\end{array}$ & $\begin{array}{l}\text { Number of water } \\
\text { particles }\end{array}$ \\
\hline 2 & 135000 & 300 & 132300 \\
Box size [-]
\end{tabular}




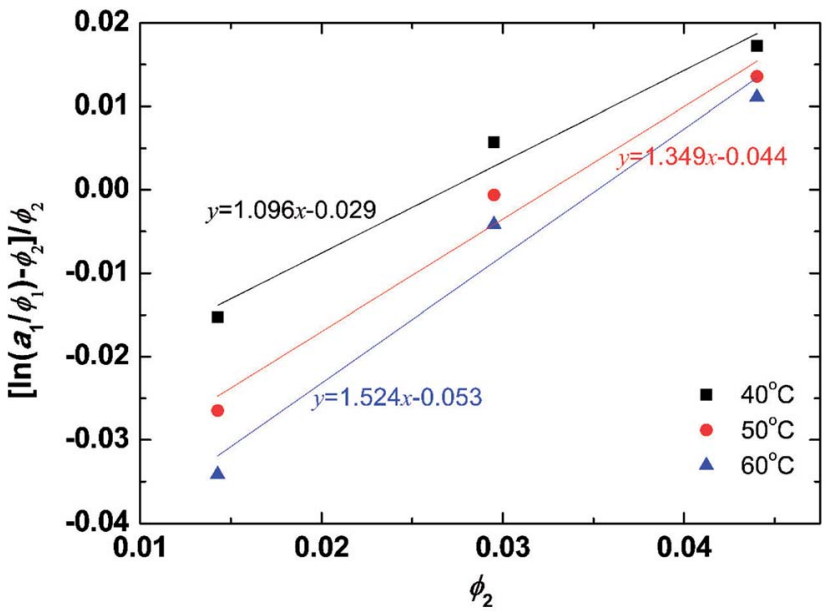

Fig. 2 Activity dependence on the PA fraction of aqueous PA solution and temperature from the Flory-Huggins theory. To confirm the validity of the fitting, we have calculated the correlation coefficient for each condition. As a result, the correlation coefficient for $40,50,60^{\circ} \mathrm{C}$ became $0.9884,0.9884$, and 0.9853 , respectively. For sample size $n=$ 3 , significance level $\alpha=0.1$ the critical value is 0.9877 , and for $n=3$, $\alpha=0.12$ the critical value is 0.9823 . Therefore, the data for 40 and $50{ }^{\circ} \mathrm{C}$ is true for a significance level of $10 \%$ and for $60{ }^{\circ} \mathrm{C}$ the correlation is true for a significance level of $12 \%$.

from spherical to worm-like micelles. At higher concentrations with $a_{\mathrm{HW}}$ exceeding 30 , the worm-like micelles spanned across the system, which resembled phase separation. These results are consistent with the works of Nakamura and Tamura, ${ }^{35}$ which reported a macroscopic phase transition of a dimer model starting from the hexagonal phase changing to the micellar phase with a complete phase separation occurring at the end. This phase transition phenomenon is similar to the lower critical solution temperature transitions reported for amphiphilic copolymers. ${ }^{4}$ Lee et al. ${ }^{17}$ also reported PA micelles changing into a branched-fiber structure across the periodic system. The phase separation found from the simulations, however, could not be observed in our PA experiments, since it was found that the C16-W3K molecules in our experiments severely degraded at temperatures around $70^{\circ} \mathrm{C}$. In terms of the

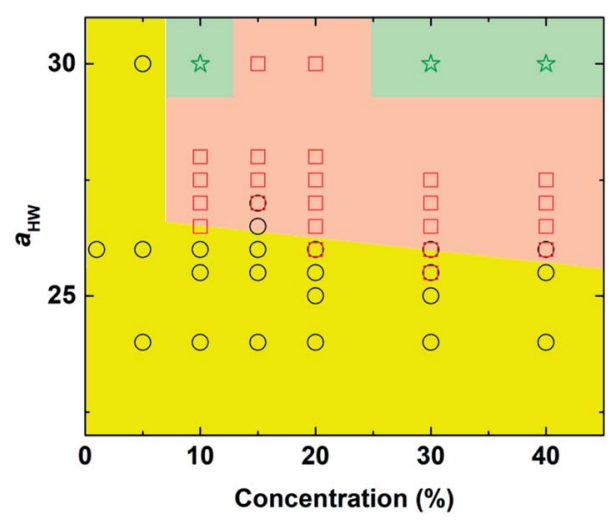

Fig. 3 Concentration- $a_{\mathrm{HW}}$ phase diagram determined by DPD simulation. Circle: spherical micelles, square: worm-like micelles, and star: phase separation (worm-like micelles spanned across the system).
$a_{\mathrm{HW}}$-dependence, it is clear from the figure that the critical concentration for the formation of worm-like micelles decreased in general as $a_{\mathrm{HW}}$ increased as seen in Fig. 3.

Snapshots taken at $t=25000 \tau$ for $a_{\mathrm{HW}}$ ranging from 10 to 30 at a concentration of $15 \%$ are presented in Fig. 4 . The hydrophilic heads and hydrophobic tails of the PA model were described as blue and red beads, respectively in the panels above, and the lower figures represent the core of the micelles elongating with increasing $a_{\mathrm{HW}}$ (temperature). Water molecules were omitted for clarity in all figures. It is clear that the size of the spherical micelles became larger as $a_{\mathrm{HW}}$ increased. The shape of the micelles transformed into more worm-like structures rather than spheres when $a_{\mathrm{HW}}$ reached 26, and worm-like micelles were eventually observable when $a_{\mathrm{HW}}$ was above 27 .

To characterize the self-assembly structure of PA more quantitatively, the asphericity of the micelles was calculated. When a coordinate system having an origin at the center-ofmass of a micelle with three principal axes of inertia is introduced, the asphericity $A$ is defined as: ${ }^{52,53}$

$$
A=\frac{\left(R_{\mathrm{g} x}{ }^{2}-R_{\mathrm{g} y}{ }^{2}\right)^{2}+\left(R_{\mathrm{g} y}{ }^{2}-R_{\mathrm{g} z}{ }^{2}\right)^{2}+\left(R_{\mathrm{g} z}{ }^{2}-R_{\mathrm{g} x}{ }^{2}\right)^{2}}{2\left(R_{\mathrm{g} x}{ }^{2}+R_{\mathrm{g} y}{ }^{2}+R_{\mathrm{g} z}{ }^{2}\right)^{2}}
$$

where $R_{\mathrm{g} x}, R_{\mathrm{g} y}$, and $R_{\mathrm{g} z}$ are the radii of gyration parallel to each principal axis.

Asphericity indicates the degree of deformation from spherical symmetry, where it is 0 for a perfect sphere and 1 for an infinite cylinder. The mean value of $A$ for all micelles forming at $t=25000 \tau$ as a function of $a_{\mathrm{HW}}$ is shown in Fig. 5 for a concentration of $15 \%$. $A$ was nearly 0 when $a_{\mathrm{HW}}$ was lower than 26.5, whereas a significant increase was observed for $a_{\mathrm{HW}}=27$, indicating a structural transition from spherical to worm-like had occurred.

To investigate the effect of $a_{\mathrm{HW}}$ on PA self-assembly over time, the mean values of $A$ for the concentration of $15 \%$ with $a_{\mathrm{HW}}$ above 27 were taken from Fig. 4 and 5, and calculated as a function of time. Fig. 6 shows the results of the time evolution of $A$. It is clear that the transition time is highly dependent on $a_{\mathrm{HW}}$. When $a_{\mathrm{HW}}$ was 30 , the transition ended 40 times faster compared to the transition at $a_{\mathrm{HW}}=27$. It is also noteworthy that the gradient of the asphericity became steeper. From these results, one can see that $a_{\mathrm{HW}}$ affects not only the eventual selfassembly structure of PA, but also the rate of self-assembly. Comparing these simulation results and our previous experimental reports, ${ }^{23,25,54}$ the $a_{\mathrm{HW}}$ dependence in simulations and temperature in experiments on the PA self-assembly coincided as expected from eqn (12). This is consistent with the findings from Shimada et al., ${ }^{55}$ where experimentally, the C16-W3K solution will transition from a spherical micellar system to a worm-like system around $50{ }^{\circ} \mathrm{C}$. This temperature corresponds to around $a_{\mathrm{HW}}=26.5$ for the DPD simulations at $15 \%$ concentration. The time evolution of the asphericity and temperature effect between the simulations and experiments are especially in accordance.

To investigate the molecular structure in more detail, intermolecular pair-distribution functions $g_{\mathrm{HW}}$ and $g_{\mathrm{HH}}$ were plotted 

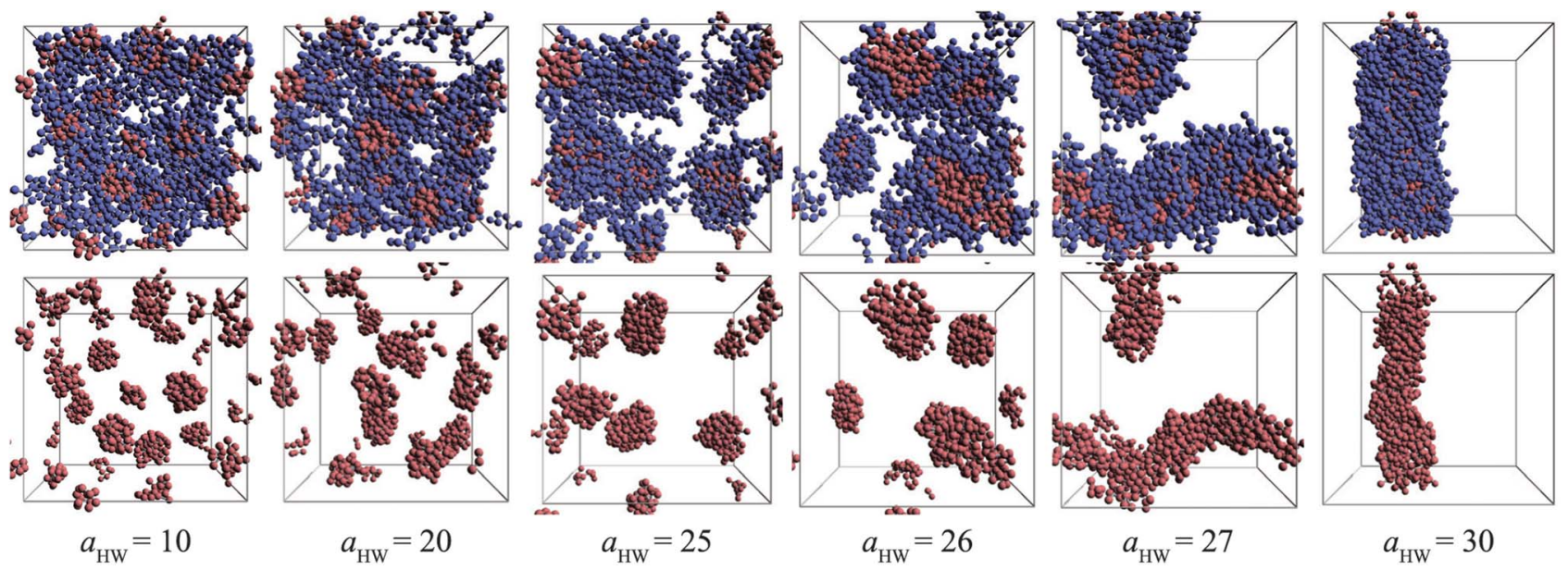

Fig. 4 Snapshots at $t=25000 \tau$ for various $a_{\mathrm{HW}}$ at concentration 15\%. The upper row shows both hydrophilic (blue) and hydrophobic (red) groups, whereas the lower row only shows the latter.

in Fig. 7 and 8, where $g_{\mathrm{HW}}$ represents the pair-distribution function for the peptides and water, and $g_{\mathrm{HH}}$ is for the intermolecular peptides. In Fig. 7, the value of $g_{\mathrm{HW}}$ stayed almost the same for $a_{\mathrm{HW}}=10$ but presented a significant decrease near the peptide as $a_{\mathrm{HW}}$ increased. This is because micelles grew with increasing $a_{\mathrm{HW}}$, thus pushing water away from the core, which was consistent with previous findings related to the hydration of peptides. ${ }^{56,57}$ Unlike Fig. 7, a sharp peak at a distance of 1 was observed as $a_{\mathrm{HW}}$ increased in Fig. 8. This peak indicates that the peptides merged at a constant distance from each other, and the amount of aggregation was proportional to the $a_{\mathrm{HW}}$ value. The variation in the packing of the peptides occurred irrespective of the interpeptide interaction parameter $a_{\mathrm{HH}}$. This is different from that found by Nakamura et al. ${ }^{35}$ where $a_{\mathrm{HH}}$ was the parameter that directly affected the packing of the amphiphiles (especially the optimal surface area of the headgroup), whereas $a_{\mathrm{HW}}$ only indirectly influenced the packing. However, in our current results, $a_{\mathrm{HW}}$ showed a significant impact on the self-assembly behavior, showing a large contribution to peptide hydration. This difference is most likely due to the fact that

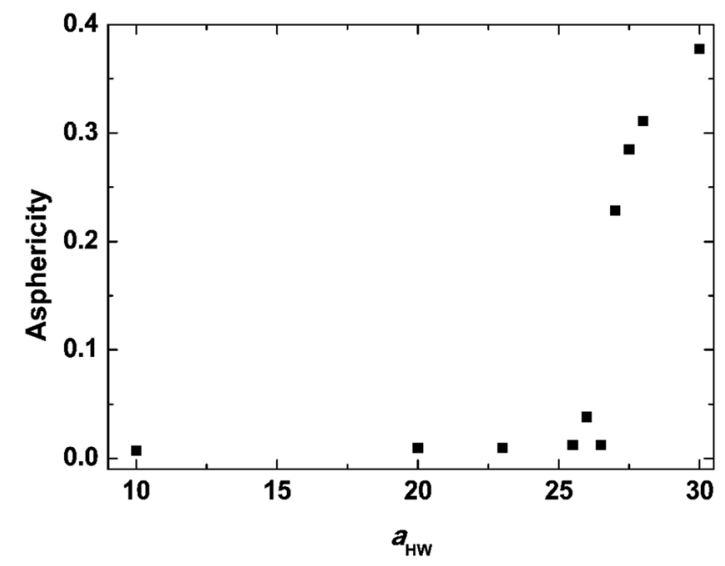

Fig. 5 Asphericity of micelles observed at $t=25000 \tau$ as a function of $a_{\mathrm{HW}}$ at concentration $15 \%$.
Nakamura et $a l .{ }^{35}$ used a non-ionic amphiphile as opposed to the zwitterionic peptide amphiphile used in this work. The fact that the difference in the innate hydrophilicities in the model changing the resultant interaction characteristics is natural.

From the simulation results, the effects of temperature, through $a_{\mathrm{HW}}$, on the mechanism of PA self-assembly can be understood. At high temperatures (with large $a_{\mathrm{HW}}$ ), the interaction between hydrophilic peptides and water became more repulsive. The relative repulsion from the peptides against water instead pulled other peptides towards themselves. Due to this attractive inter-peptide force, spherical micelles could easily gather closely, so they could fuse into worm-like micelles. Fast progress of phase transitions at high temperatures can be considered as a result of a higher probability of micelle fusion. On the other hand, at low temperatures, which could be represented by a low $a_{\mathrm{HW}}$ value, the interaction between hydrophilic peptides and water became relatively attractive. Therefore, water was distributed stably around the hydrated peptides, suppressing the aggregation of spherical micelles without further transitions into worm-like micelles. Such a slow progress of the phase transition at low temperatures could be regarded as a result of the low probability of micelle fusion.

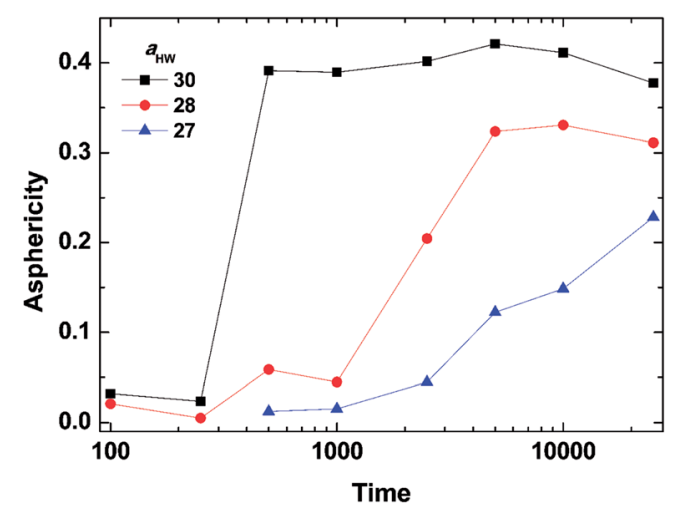

Fig. 6 Asphericity evolution of micelles at concentration 15\%. The lines are there to guide the eye. 


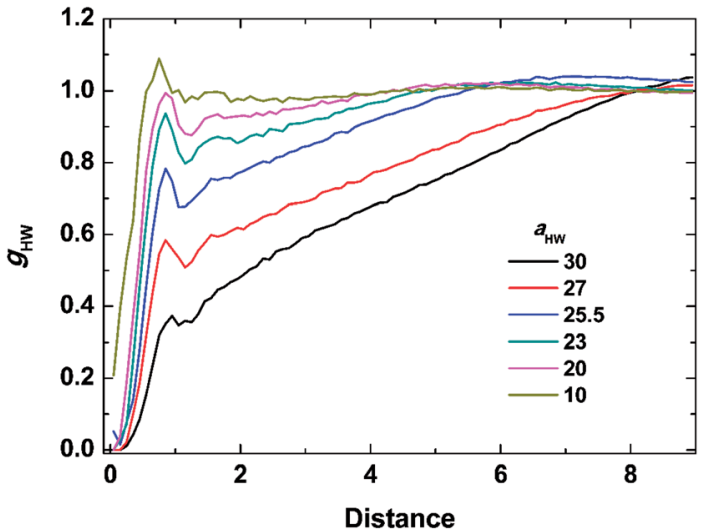

Fig. 7 Pair distribution function for the peptides and water at concentration $15 \%$ and $t=25000 \tau$.

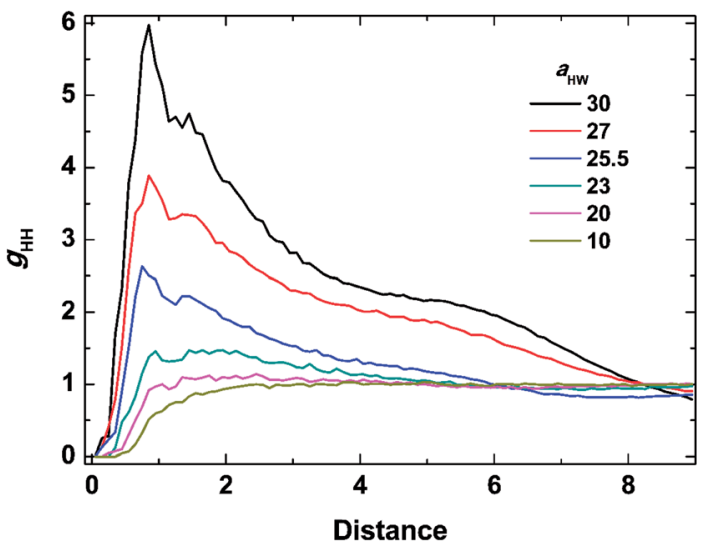

Fig. 8 Pair distribution function for inter-molecular peptides at concentration $15 \%$ and $t=25000 \tau$.

\section{Conclusion}

VPO measurements were conducted prior to DPD calculations to model the interaction parameters to simulate the selfassembly of PA dissolved in an aqueous solution. In terms of the Flory-Huggins theory, the interaction between the peptides and water was confirmed to be more repulsive as the temperature increased. The dynamics of the self-assembly and resulting microstructures of PA revealed to be highly dependent on the value of the repulsion parameter $a_{\mathrm{HW}}$ by DPD simulation, and the relation between temperature and repulsion parameter $a_{\mathrm{HW}}$ was clarified. The mechanism of temperature dependence on the phase transition of PA starts from water molecules hydrating the hydrophilic head groups at low temperature, leading to a low probability of micelle fusion. On the other hand, the interaction between hydrophilic head groups became relatively attractive at high temperatures, leading to a high probability of micelle fusion and thereafter worm-like transition.

The primary link between simulation and experiment in this study does not lie in conventional parametric fitting, but rather in attaining an understanding in the thermosensitivity of interactions between peptide amphiphiles in an aqueous solution through both methods simultaneously. The relation between an increase in temperature (experimental) and increase in hydrophobicity has become clear (computational). Based on this finding, we confirmed that $a_{\text {HW }}$ plays an important role in self-assembly characteristics. The methodology in quantitatively linking the coarse-grained parameters from experiment and theory provides a sensible foundation for bridging future simulation studies with experimental work on macromolecules.

\section{Conflicts of interest}

There are no conflicts of interest to declare.

\section{Acknowledgements}

N. A. was supported by JSPS KAKENHI Grant number 17K14610.

\section{References}

1 I. Dimitrov, B. Trezebicka, A. H. E. Muller, A. Dworak and C. B. Tsvetanov, Prog. Polym. Sci., 2007, 32, 1275-1343.

2 L. E. Bromberg and E. S. Ron, Adv. Drug Delivery Rev., 1998, 31, 197-221.

3 C. Gong, S. Shi, P. Dong, B. Kan, M. Gou, X. Wang, X. Li, F. Luo, X. Zhao, Y. Wei and Z. Qian, Int. J. Pharm., 2009, 365, 89-99.

4 Y. Zhou, D. Yan, W. Dong and Y. Tian, J. Phys. Chem. B, 2007, 111, 1262.

5 Y. H. Lim, D. Kim and D. S. Lee, J. Appl. Polym. Sci., 1997, 64, 2647.

6 Y. Tanaka, Y. Kagami, A. Matsuda and Y. Osada, Macromolecules, 1995, 28, 2574-2576.

7 H. G. Cui, M. J. Webber and S. I. Stupp, Biopolymers, 2010, 94, $1-18$.

8 X. B. Zhao, F. Pan, H. Xu, M. Yaseen, H. H. Shan, C. A. E. Hauser, S. G. Zhang and J. R. Lu, Chem. Soc. Rev., 2010, 39, 3480-3498.

9 S. C. Song, S. B. Lee, J. I. Jin and Y. S. Sohn, Macromolecules, 1999, 32, 2188-2193.

10 J. Y. Kim, M. H. Park, M. K. Joo, S. Y. Lee and B. Jeong, Macromolecules, 2009, 42, 3147-3151.

11 J. J. Panda, A. Mishra, A. Basu and V. S. Chauhan, Biomacromolecules, 2008, 9, 2244-2250.

12 A. P. Nowak, V. Breedveld, L. Pakstis, B. Ozbas, D. J. Pine, D. Pochan and T. J. Deming, Nature, 2002, 417, 424.

13 S. Q. Liu, P. L. Rachel Ee, C. Y. Ke, J. L. Hedrick and Y. Y. Yang, Biomaterials, 2009, 30, 1453-1461.

14 H. Yokoi, T. Kinoshita and S. Zhang, Proc. Natl. Acad. Sci. U. S. A., 2005, 102, 8414-8419.

15 W. Ding, S. Lin, J. Lin and L. Zhang, J. Phys. Chem. B, 2008, 112, 776-783.

16 O. S. Lee, S. I. Stupp and G. C. Schatz, J. Am. Chem. Soc., 2011, 133, 3677-3683.

17 O. S. Lee, V. Cho and G. C. Schatz, Nano Lett., 2012, 12, 49074913. 
18 I. W. Fu, C. B. Markegard, B. K. Chu and H. D. Nguyen, Langmuir, 2014, 30, 7745-7754.

19 OCTA home page, https:/www.octa.jp/OCTA/cognac.html, accessed June 1st 2018.

20 S. O. Yesylevskyy, L. V. Schäfer, D. Sengupta and S. J. Marrink, PLoS Comput. Biol., 2010, 6, e1000810.

21 I. W. Fu, C. B. Markegard, B. K. Chu and H. D. Nguyen, Adv. Healthcare Mater., 2013, 2, 1388-1400.

22 A. V. Smith and C. K. Hall, Proteins, 2001, 44, 376-391.

23 T. Shimada, S. Lee, F. S. Bates and A. Hotta, J. Phys. Chem. B, 2009, 113, 13711-13714.

24 T. Shimada and M. Tirrell, Abstracts of Papers: 230th ACS National Meeting, 2005, vol. 230, pp. U3537-U3538.

25 T. Shimada, A. Hotta and M. Tirrell, Polym. Mater. Sci. Eng., 2006, 93, 127-128.

26 C. Duce, S. Monti, R. Solaro and M. R. Tine, J. Phys. Chem. B, 2007, 111, 1165-1175.

27 P. J. Hoogerbrugge and J. M. V. A. Koelman, Europhys. Lett., 1992, 19, 155-160.

28 J. Koelman and P. J. Hoogerbrugge, Europhys. Lett., 1993, 21, 363-368.

29 R. D. Groot and P. B. Warren, J. Chem. Phys., 1997, 107, 44234435.

30 E. Ryjkina, H. Kuhn, H. Rehage, F. Muller and J. Peggau, Angew. Chem., Int. Ed., 2002, 41, 983-986.

31 H. Nakamura and Y. Tamura, J. Plasma Phys., 2006, 72, 10011004.

32 S. Jury, P. Bladon, M. Cates, S. Krishna, M. Hagen, N. Ruddock and P. Warren, Phys. Chem. Chem. Phys., 1999, 1, 2051-2056.

33 N. Arai, K. Yasuoka and Y. Masubuchi, J. Chem. Phys., 2007, 126, 244905.

34 N. Arai, K. Yasuoka and X. C. Zeng, ACS Nano, 2016, 10, 8026-8037.

35 H. Nakamura and Y. Tamura, Comput. Phys. Commun., 2005, 169, 139-143.

36 A. Eliassi, H. Modarress and G. A. Mansoori, J. Chem. Eng. Data, 1999, 44, 52-55.
37 M. Karimi, W. Albrecht, M. Heuchel, T. Weigel and A. Lendlein, Polymer, 2008, 49, 2587-2594.

38 R. Dickman and C. K. Hall, J. Chem. Phys., 1986, 85, 41084115.

39 P. Español and P. Warren, Europhys. Lett., 1995, 30, 191-196. 40 S. Marqusee, V. H. Robbins and R. L. Baldwin, Proc. Natl. Acad. Sci. U. S. A., 1989, 86, 5286-5290.

41 P. G. M. Wuts and T. W. Greene, Greene's Protective Groups in Organic Synthesis, John Wiley \& Sons Inc, 5th edn, 2014.

42 M. Doi, Macromol. Symp., 2003, 195, 101-108.

43 L. Gao, J. Shillcock and R. Lipowsky, J. Chem. Phys., 2007, 126, 015101.

44 X. Song, P. Shi, M. Duan, S. Fang and Y. Ma, RSC Adv., 2015, 5, 62971-62981.

45 Y. Ma, Y. Wang, X. Deng, G. Zhou, S. Khalid, X. Sun, W. Sun, Q. Zhou and G. Lu, RSC Adv., 2017, 7, 39676-39684.

46 S. Yamamoto and S. Hyodo, J. Chem. Phys., 2005, 122, 204907.

47 S. Yamamoto and S. Hyodo, Polym. J., 2003, 35, 519-527.

48 N. Arai, Y. Yoshimoto, K. Yasuoka and T. Ebisuzaki, Phys. Chem. Chem. Phys., 2016, 18, 19426-19432.

49 Y. Kobayashi and N. Arai, RSC Adv., 2018, 8, 18568-18575.

50 R. D. Groot and T. J. Madden, J. Chem. Phys., 1998, 108, 87138724.

51 R. D. Groot and K. L. Rabone, Biophys. J., 2001, 81, 725-736. 52 B. Capone, C. Pierleoni, J. P. Hansen and V. Krakoviack, J. Phys. Chem. B, 2009, 113, 3629-3638.

53 S. Fujiwara, T. Itoh, M. Hashimoto and Y. Tamura, Mol. Simul., 2007, 33, 115-119.

54 T. Shimada, K. Megley, M. Tirrell and A. Hotta, Soft Matter, 2011, 7, 8856-8861.

55 T. Shimada, N. Sakamoto, R. Motokawa, S. Koizumi and M. Tirrell, J. Phys. Chem. B, 2012, 116, 240-243.

56 M. Vogel, J. Phys. Chem. B, 2009, 113, 9386-9392.

57 P. Mark and L. Nilsson, J. Phys. Chem. A, 2001, 105, 99549960. 